\title{
El REgReso de ORTEGA: los PRIMEROS PASOS DE SU SEGUNDA ADMINISTRACIÓN
}

\author{
The Return of Ortega: The First Steps of his Second Administration
}

\section{SALVADOR MARTÍ I PUIG}

Universidad de Salamanca

\begin{abstract}
RESUMEN
El año 2007 en Nicaragua estuvo marcado por la llegada de Daniel Ortega a la Presidencia de la República y por las primeras medidas de su nueva administración. Centrándose en dicho acontecimiento el texto que se presenta razona, en primer lugar, por qué el FSLN llegó nuevamente al poder. Posteriormente analiza la composición y naturaleza del nuevo gobierno, así como las primeras medidas implementadas. A continuación presenta la dinámica de coaliciones y pactos que se sucedieron en la Asamblea Nacional, donde el FSLN es la minoría mayor; y señala el debate de reforma constitucional en curso. Finalmente el artículo concluye con un análisis de la mutación realizada por el FSLN desde que perdió las elecciones en 1990 hasta que recuperó el poder en 2007, y en base a ésta plantea qué puede esperarse de la segunda Administración Ortega.
\end{abstract}

Palabras clave: Nicaragua, Sandinismo, FSLN, Daniel Ortega, Policy Change, Pactos.

\begin{abstract}
In 2007 Nicaragua saw the electoral victory of Daniel Ortega to the presidential office and the first policy decisions of his government. This article first examines the process that brought the FSLN back to power. Secondly, it analyzes the composition and nature of the new government, as well as its first steps taken. It then presents the dynamics of the pacts and coalitions which have formed successively in the National Assembly, where the FSLN holds the largest minority position, and discusses the current constitutional reform debate. Finally, the article concludes with an analysis of the process of transformation that took place within the FSLN since it lost the election of 1990 until its return to power in 2007. On the basis of this change it asks what may be expected from the second Ortega administration.
\end{abstract}

Key words: Nicaragua, Sandinism, FSLN, Daniel Ortega, Policy Change, Pacts.

\section{I. ¿CÓMO FUE POSIBLE LA VICTORIA SANDINISTA?}

Los resultados de las elecciones de 2006 dieron al traste al formato de competencia partidaria que existía en elecciones de carácter nacional desde 1990, donde el FSLN y una coalición "antisandinista" (que siempre fue mayoritaria) competían por escaños en la Asamblea Nacional y por la Presidencia de la República. En 2006 el candidato del FSLN Daniel Ortega ganó la máxima magistratura y su partido obtuvo la mayoría simple en la Asamblea Nacional -tal como se muestra en el Anexo 1. 
Ante dichos resultados la pregunta pertinente es: ¿Cuáles fueron los factores que explican la victoria del FSLN? Para responder dicha cuestión es preciso señalar cuatro elementos: El pacto establecido entre Daniel Ortega y Arnoldo Alemán, un realineamiento electoral que transformó la dinámica presente en la arena política desde 1990, las características organizativas del FSLN, y el discurso de campaña que elaboraron los sandinistas.

El pacto a que nos referimos (EP desde ahora) es el acuerdo que en enero del año 2000 sellaron el entonces Presidente de la República Arnoldo Alemán y Daniel Ortega para hacerse con el control bipartidista (del PLC y el FSLN) de las instituciones clave del Estado (entre las que destaca la Oficina del Contralor General de la República, la Corte Suprema de Justicia y el Consejo Supremo Electoral) y para cambiar la Ley Electoral en beneficio de ambos caudillos. ${ }^{1}$ La nueva Ley Electoral supuso que se podía ganar el cargo de Presidente de la República con el $40 \%$ de los votos o con un 35\% del sufragio si la diferencia entre los dos candidatos mejor situados era superior al $5 \% .{ }^{2}$ Fue esta nueva "fórmula" la que hizo posible que el día 5 de noviembre de 2006 Daniel Ortega obtuviera la máxima magistratura con un 38\% del sufragio a pesar de ser el candidato que mayor rechazo generaba entre la población.

El realineamiento en la arena política a que hacemos referencia fue el fruto del posicionamiento de las fuerzas políticas respecto a $\mathrm{EP}$, fenómeno que supuso la desaparición de la dinámica bipoplar caracterizó la competencia electoral desde 1990 -a saber, la del sandinismo vs antisandinismo. ${ }^{3}$ Así, los votos dejaron de concentrarse sólo en dos opciones para hacerlo en cuatro: el FSLN (sandinismo pro pacto), el PLC (liberalismo pro pacto), la ALN (liberalismo antipacto) y el MRS (sandinismo antipacto). Esta fragmentación favoreció al FSLN que contaba con un voto fiel y duro. ${ }^{4}$ Ese fenómeno supuso que, con la Ley Electoral vigente antes descrita, Daniel Ortega ganara la Presidencia. Contrariamente, el liberalismo, que concurrió en 2006 a través de dos opciones enfrentadas dividió su voto casi por la mitad (el $26 \%$ para el PLC y el $29 \%$ para la ALN) y no hubo posibilidad de ballotage.

Respecto a las características organizativas del FSLN cabe destacar, en primer lugar, que los sandinistas disponían una máquina partidaria muy bien engrasada, con un tendido territorial nacional muy denso y con la propiedad de canales de televisión y de radios. Y en segundo lugar, por la disciplina orgánica y la unión incondicional de todos los militantes sandinistas alrededor de la figura de Daniel Ortega. ${ }^{5}$

Para mayor información sobre EP véase: (Dye, 2001, 2004; Close 2004; Martí 2007; Pérez Baltodano 2005).

Se trata de la Ley $n^{\circ} 331$, aprobada el 10 de enero de 2000 y publicada en la Gaceta $n^{\circ} 16$ del 24 de enero del mismo año.

3 Esta es la hipótesis que se desarrolla en el trabajo de Martí (2008), donde se analiza el desenlace electoral de las pasadas elecciones de 2006 haciendo énfasis en la literatura sobre realineamientos y dealineamientos electorales, con una especial atención a los trabajos seminales de Key $(1955,1964)$ y los desarrollos posteriores de Carmines et al. (1987) Carmines y Wagner (2006) y Warren (1991).

4 Con todo, si se divide el resultado electoral en bloques de izquierda (sumando los sufragios del FSLN y el MRS) y derecha (juntando los resultados del PLC y ALN) constatamos que la izquierda sólo conquistó cinco de los 17 departamentos. En el resto del país la hegemonía del voto fue claramente "liberal". En la revista Envío del mes de diciembre de 2006 (www.envio.org) se puede observar un exhaustivo trabajo de análisis electoral, contrastando el voto departamentos, la adjudicación de diputados y comparando los resultados del 2006 con los del 2001.

5 Sobre esta cuestión ver el trabajo de Ortega Hegg (2006). 
Finalmente, en cuanto a los "recursos discursivos", es preciso anotar el despliegue por parte del FSLN de una larga e intensa campaña que prometía "paz y amor" y que decía que "todo se podía solucionar". La nueva política del FSLN (que en la convocatoria de 2006 se presentó bajo el eslogan Gran Alianza Nicaragua Triunfa) elaboró un discurso basado en un mensaje de "amor, reconciliación y perdón", además de un nutrido abanico de promesas que iban desde "una política masiva de créditos y subsidios para todos los productores; la condonación de las deudas a todos los acreedores, la regulación de las agencias bancarias que canalizan las remesas de los emigrantes, y hasta petróleo venezolano (barato) para abastecer a Nicaragua" (Ortega Hegg, 2006). Con ello, la campaña sandinista evitó hablar de conflicto y de clases sociales y prefirió elaborar una dinámica festiva y despolitizada. ${ }^{6}$ Finalmente también cabe señalar el cambio realizado por parte del FSLN a la hora de tratar temas de carácter moral, como es el del aborto terapéutico, donde éste se alineó con sectores de la Iglesia Católica con los que años antes se había enfrentado.

Pero más allá de la victoria sandinista los resultados de los comicios de 2006 dieron cuenta de un país territorialmente dividido entre sandinistas y antisandinistas, y sobre todo, de un liberalismo también territorialmente dividido entre los modernizadores" representados por ALN y los "tradicionalistas y populistas" (mayoritariamente rurales) vinculados al PLC.

Así, Nicaragua quedó conformada en un país con tres regiones políticamente diferenciadas. Por un lado, apareció el eje urbano de Managua-León-Chinandega, el norte cafetalero y la Región Autónoma del Atlántico Norte (donde el FSLN pactó con la formación indigenista Yatama) de ascendencia sandinista. Por otro, la región sur occidental (el oriente), relativamente desarrollada por el turismo y por la cercanía con Costa Rica que apoyó al "proyecto neoliberal" de oportunidades que pregonaba la ALN. Y finalmente, una región "interior" de economía campesina y ganadera donde el liberalismo "tradicional y populista" del PLC es mayoritario.?

Pero a pesar de la ajustada victoria del FSLN, el desempeño de Daniel Ortega durante el 2007 no fue precisamente discreto ni celoso en la búsqueda de consensos. En este sentido, los fastos de su toma de posesión como Presidente de la República el día 10 de enero (en la que participaron diversos Jefes de Estado y fue presenciada por 250.000 personas $)^{8}$ mostraron el estilo personalista de su nueva administración. Una administración que dio lugar a muchas especulaciones debido a los temores y las expectativas que despertó, ya que ésta podía suponer una ruptura al "ciclo" de tres quinquenios seguidos de políticas neoliberales y pro norteamericanas. Responder hasta qué punto el segundo gobierno

6 Respecto a la pérdida de radicalidad y la ausencia del conflicto en el discurso del FSLN durante la campaña electoral de 2006 véase el texto de Pérez Baltodano (2006).

7 Además, cabe apuntar que de los 17 departamentos del país, el FSLN siempre ganó o quedó segundo (con la excepción de Chontales y la Región Autónoma del Atlántico Sur), mientras que ALN y PLC se dividieron territorialmente el voto, compitiendo entre sí, quedando en la primera o tercera posición según los, tal como se observa en la Tabla 3 que aparece en el anexo.

8 En la ceremonia destacó la presencia de los Jefes de Estado de México, Ecuador, Panamá, Honduras, Colombia, Taiwán, Haití, Irán y, sobre todo, los de Venezuela y Bolivia, Hugo Chávez y Evo Morales, que se sentaron a cada lado de Ortega. 
Ortega supuso un policy change o una simple "circulación" de elites en el poder es uno de los motivos de este texto.

\section{NUEVO GOBIERNO: ¿NUEVAS POLÍTICAS Y ESTILOS?}

Las promesas realizadas por Daniel Ortega durante la larga campaña electoral de 2006 fueron muy diferentes a las que el mismo dirigente hizo en 1984 y en 1990. En 1984, en medio de una revolución y una guerra, el líder sandinista prometió "gallo pinto y dignidad", ${ }^{9}$ sin embargo en la campaña de 2006 Ortega cambió de discurso, y en una estrategia expansiva ofertó "una política masiva de créditos y subsidios para todos los productores, la condonación de las deudas a todos los acreedores, la regulación de las agencias bancarias que canalizan las remesas de los emigrantes, y petróleo venezolano para abastecer a Nicaragua".

Así las cosas, durante las primeras semanas de 2007 reinó la expectación sobre qué tipo de gobierno y qué tipo de políticas públicas se diseñarían. Ciertamente las expectativas generadas demandaban un gobierno proactivo y un notable policy change para satisfacer una parte de la clientela electoral sandinista sedienta de políticas sociales después de tres lustros con un gasto social público prácticamente inexistente (Spalding, en prensa).

Respecto al tipo de gobierno, Ortega diseñó un gabinete de bajo perfil y con rol preeminente de su esposa -la Primera Dama Rosario Murillo. En dicho gabinete no apareció ningún cuadro histórico (ni relevante) del sandinismo de los años ochenta, ni del período en que el partido estuvo en la oposición. Los tres cuadros partidarios más importantes que aún pertenecían al FSLN (con excepción de Ortega) no tuvieron ninguna relevancia en el nuevo Ejecutivo. Tomás Borge, fundador del FSLN y vicesecretario general del partido, fue "destinado" a Perú como Embajador de Nicaragua; Bayardo Arce, también Comandante de la Revolución y gestor del patrimonio del FSLN tampoco tuvo ningún cargo ejecutivo; y Lenín Cerna ex miembro de la inteligencia del Ministerio del Interior, cuadro del Ejército Nacional y responsable hasta 2006 de la organización interna del FSLN fue desplazado por la Primera Dama. Así las cosas, la mayoría de ministros designados fueron miembros sandinistas con una limitada proyección pública y, por lo tanto, con una débil posición frente al Presidente de la República, ${ }^{10}$ con la excepción del Cardenal Obando y Bravo (quien durante los años ochenta fue un furibundo enemigo de la revolución y del FSLN) que fue nombrado Presidente de la "Comisión Interinstitucional para la reconciliación, paz y justicia" con rango ministerial. La tabla que sigue expone la relación de los ministros de la administración Ortega y sus características.

9 Gallo pinto es una comida popular que consta de frijoles y arroz refrito y que es base de la dieta de la mayoría de los nicaragüenses. Sobre dicha promesa y las campañas de 1984 y 1990 ver: Martí, 1997.

10 Por ello se explica también la inestabilidad gubernamental y la rotación de cargos en algunos ministerios, siendo el caso más alarmante el cambio de tres ministros durante el año en los Ministerios de Salud, Cultura y Medio Ambiente. Por esta misma razón la tabla que se expone puede perder vigencia con una relativa celeridad. 
Tabla 1: Composición del gobierno y la relación de los ministros con el FSLN

\begin{tabular}{|c|c|c|c|}
\hline Ministerio & Titular y perfil partidario & $\begin{array}{l}\text { Relación de los ministros } \\
\text { con el Presidente y la } \\
\text { Primera Dama }\end{array}$ & Observaciones \\
\hline Salud & $\begin{array}{l}\text { Guillermo González } \\
\text { Bajo perfil partidario }\end{array}$ & $\begin{array}{l}\text { Relación directa con la } \\
\text { Primera Dama. }\end{array}$ & $\begin{array}{l}\text { Uno de los ministerios } \\
\text { con mayor circulación de } \\
\text { titulares. }\end{array}$ \\
\hline Agropecuario y Forestal & $\begin{array}{l}\text { Ariel Bucardo } \\
\text { Procede de la Unión } \\
\text { Nacional de Ganaderos y } \\
\text { Agricultores, grupo afín } \\
\text { al FSLN. }\end{array}$ & $\begin{array}{l}\text { Relación directa con } \\
\text { Daniel Ortega y su asesor } \\
\text { en políticas sociales, } \\
\text { Orlando Núñez. }\end{array}$ & \\
\hline Educación & $\begin{array}{l}\text { Miguel de Castilla. } \\
\text { Académico, bajo perfil } \\
\text { partidario. }\end{array}$ & $\begin{array}{l}\text { Relación directa con } \\
\text { Daniel Ortega y la } \\
\text { Primera Dama. }\end{array}$ & \\
\hline Defensa & $\begin{array}{l}\text { Ruth Tapia Roa } \\
\text { Cuadro medio del FSLN. }\end{array}$ & $\begin{array}{l}\text { Relación directa con } \\
\text { Daniel Ortega. }\end{array}$ & \\
\hline Gobernación & $\begin{array}{l}\text { Ana Isabel Morales } \\
\text { Cuadro medio del } \\
\text { FSLN, ex miembro de } \\
\text { la Seguridad del Estado } \\
\text { durante la revolución. }\end{array}$ & $\begin{array}{l}\text { Relación directa con la } \\
\text { Primera Dama. }\end{array}$ & $\begin{array}{l}\text { No es cercana a Lenín } \\
\text { Cerna, ex responsable } \\
\text { de la Seguridad del } \\
\text { Estado y Secretaria de } \\
\text { Organización del FSLN. }\end{array}$ \\
\hline $\begin{array}{l}\text { Hacienda y Crédito } \\
\text { Público }\end{array}$ & $\begin{array}{l}\text { Alberto Guevara } \\
\text { Bajo perfil partidario }\end{array}$ & $\begin{array}{l}\text { Relación directa con } \\
\text { con Bayardo Arce, } \\
\text { responsable de finanzas } \\
\text { del FSLN. }\end{array}$ & \\
\hline $\begin{array}{l}\text { Familia, Adolescencia y } \\
\text { Niñez }\end{array}$ & $\begin{array}{l}\text { Rosa Afilia Vizcaya. Bajo } \\
\text { perfil partidario. }\end{array}$ & $\begin{array}{l}\text { Relación directa con la } \\
\text { Primera Dama. }\end{array}$ & \\
\hline Relaciones Exteriores & $\begin{array}{l}\text { Samuel Santos. } \\
\text { Exsecretario } \\
\text { de relaciones } \\
\text { internacionales del } \\
\text { FSLN. }\end{array}$ & $\begin{array}{l}\text { Relación directa con } \\
\text { Daniel Ortega y también } \\
\text { con Bayardo Arce. }\end{array}$ & $\begin{array}{l}\text { Cuenta con el apoyo del } \\
\text { Padre D'Escoto, figura } \\
\text { de confianza de Daniel } \\
\text { Ortega en relaciones } \\
\text { internacionales. }\end{array}$ \\
\hline $\begin{array}{l}\text { Transporte e } \\
\text { Infraestructura }\end{array}$ & $\begin{array}{l}\text { Fernando Martínez. Bajo } \\
\text { perfil partidario }\end{array}$ & $\begin{array}{l}\text { Relación directa con la } \\
\text { Primera Dama. }\end{array}$ & \\
\hline Medio Ambiente & $\begin{array}{l}\text { Juanita Argeñal } \\
\text { Bajo perfil partidario }\end{array}$ & $\begin{array}{l}\text { Relación directa con la } \\
\text { Primera Dama. }\end{array}$ & \\
\hline $\begin{array}{l}\text { Comisión de } \\
\text { Reconciliación y Paz }\end{array}$ & $\begin{array}{l}\text { Miguel Obando y Bravo } \\
\text { Cardenal }\end{array}$ & $\begin{array}{l}\text { Relación directa con } \\
\text { Daniel Ortega y la } \\
\text { Primera Dama. }\end{array}$ & $\begin{array}{l}\text { Es el sacerdote que ofició } \\
\text { el matrimonio católico } \\
\text { entre Daniel Ortega y } \\
\text { Rosario Murillo. }\end{array}$ \\
\hline
\end{tabular}

Fuente: elaboración propia a partir de la información de las revistas Envío y Confidencial.

En este diseño gubernamental, con una amplia intermediación de la Primera Dama, también cabe añadir que el norteamericano naturalizado nicaragüense y antiguo colaborador de Ortega durante la década de los ochenta, Paul Oquist, ejerció de "hombre fuerte" en tanto que Secretario de la Presidencia. En este contexto tanto el mencionado Secretario de la Presidencia 
como los "asesores directos" del Presidente en temas sectoriales (como desarrollo económico, políticas sociales o relaciones internacionales) tuvieron mayor poder que los mismo Ministros "de línea" quienes ejercieron un rol de meros ejecutores con una limitadísima capacidad de manejar la agenda y de expresar sus opiniones en público y cuya continuidad dependía mucho de la relación directa con el Presidente y la Primera Dama.

Respecto a las políticas públicas, las primeras señales de Ortega al frente de la Presidencia fueron contradictorias: Por un lado anunció un "giro social" en el ámbito de las políticas sociales pero, por el otro, mostró -a pesar de la retórica antiimperialista formulada en los foros internacionales- una total continuidad respecto a las políticas macroeconómicas y comerciales.

El anunciado "giro social" se basó en dos ejes. El primero de ellos fue el despliegue de políticas sociales focalizadas para paliar la pobreza a través de programas sectoriales llamados Hambre Cero, Usura Cero y Desempleo Cero. ${ }^{11}$ El segundo fue decretar la gratuidad total de la educación primaria y secundaria, y de la atención de centros de salud y hospitales. Sin embargo, las medidas de "gratuidad de servicios" se realizaron sin una dotación adecuada de fondos públicos que, junto con la prohibición a los profesionales de cobrar a los usuarios, generaron un amplio descontento entre los trabajadores de la educación y de la salud, quienes emprendieron huelgas que se extendieron durante todo el primer trimestre de la administración sandinista, creando el primer gran conflicto laboral de la administración Ortega. ${ }^{12}$

La continuidad de políticas a que hacemos referencia se constató por el mantenimiento de la política macroeconómica y comercial, y por la misma voluntad que las administraciones anteriores de captar inversiones de capital extranjero en condiciones sumamente favorables para este último. No en vano la primera visita de Ortega como Jefe de Estado fue en San Salvador con el magnate mexicano (y ahora la persona más rica del mundo) Carlos Slim, al que le ofreció un trato exquisito en el caso de invertir en Nicaragua. En esta dirección la política macroeconómica impulsada por Ortega -quien expuso en la Asamblea Nacional a la hora de defender los presupuestos de 2008 que tenía "un corazón de izquierda justiciero, y una cabeza de derecha reponsable" $1{ }^{13}$ no se diferenció de la implementada por las tres anteriores administraciones liberales. Efectivamente, el diseño de los presupuestos de

11 De todos los programas, el más publicitado fue el de Hambre Cero, compuesto por un Bono Productivo junto con dos subprogramas, uno destinado a la erradicación de la desnutrición crónica infantil y otro de merienda escolar. El costo total del programa, según cifras oficiales, es el 150.000 millones de dólares y el número de beneficiarios durante la administración asciende a 15.000 familias. En un inicio el director del programa era el sociólogo y director de la ONG llamada CIPRES, afín al FSLN, Orlando Núñez, pero a los pocos meses Núñez fue destituido y pasó a ser "asesor presidencial" en temas de políticas sociales y el mismo programa perdió centralidad. Por otro lado Usura Cero es un programa de préstamo "blando" destinado a zonas urbanas (especialmente a pequeños comerciantes), con créditos que oscilan entre 1.850 y 5.500 córdobas. En 2007 este programa se inició tímidamente y aún no existe perspectiva para poder ver sus efectos. Sobre el programa Desempleo Cero aún hay poca información disponible.

12 Los conflictos sociales más relevantes del año fueron la huelga de maestros (que duró un mes) y la de médicos. En la huelga de médicos (en que el sindicato sandinista FETSALUD no apoyó a los movilizados) los profesionales de la salud pedían el cumplimiento del convenio laboral. La huelga de maestros, que duró un mes, también hubo reivindicaciones salariales y se terminó con un frágil acuerdo entre los maestros y el Ministerio de Hacienda y el Seguro Social. Otra huelga a destacar (llamanda de Nemagon) la realizaron ex trabajadores de las bananeras que reclamaban indemnizaciones y salud gratuita.

13 Extraído de Envío, número de noviembre 2007. 
2008 fue muy semejante al de años anteriores, especialmente respecto al pago de la deuda interna y a la ausencia de una política fiscal progresiva. Y en cuanto al resultado de las negociaciones con el FMI también fue muy parecido al de años anteriores.

Con todo, la combinación de las declaraciones beligerantes de Ortega en los foros internacionales, ${ }^{14}$ junto con el cambio de interlocutores internacionales ${ }^{15}$ (sin dejar de pertenecer al CAFTA) y la ausencia de una agenda política claramente definida (hecho que generó una notable parálisis gubernamental en los ministerios) ${ }_{1}^{16}$ incrementó la incertidumbre sobre el futuro de la economía del país.

Los datos disponibles para el año 2007 no fueron demasiado satisfactorios. El crecimiento osciló entre un 3,7\% a 3,9\% del PIB, inferior al 4,2\% acumulado de la anterior administración Bolaños; a la par que la producción de maíz y frijol cayó en un 8,7 y 4,2\% respectivamente. A ello se le sumó una inflación del 10,71\% para el mes de octubre, que golpeó sobre todo a la canasta básica con un 24,8 \%, sólo superada por el precio del petróleo, que subió un 25,6\%. ${ }^{17}$ Así, según el Banco Central de Nicaragua, la inflación de 2007 llegó a 16,8, siete puntos más que los últimos tres años del gobierno Bolaños y la más elevada del resto de países de la región -tal como puede observarse en la Tabla 2 que sigue.

Tabla 2: Inflación acumulada anual (\%)

\begin{tabular}{lrrrrrrrr}
\hline País & 2000 & 2001 & 2002 & 2003 & 2004 & 2005 & 2006 & 2007 \\
\hline Nicaragua $^{1}$ & 10,17 & 4,84 & 3,87 & 6,48 & 9,26 & 9,58 & 9,45 & 16,8 \\
Costa Rica $^{2}$ & 10,25 & 10,96 & 9,68 & 9,87 & 13,13 & 14,07 & 9,43 & 10,81 \\
Honduras $^{3}$ & 10,1 & 8,8 & 8,1 & 6,8 & 9,2 & 7,7 & 5,3 & 8,9 \\
El Salvador $^{4}$ & 4,3 & 1,4 & 2,8 & 2,5 & 5,4 & 4,3 & 4,9 & 4,9 \\
Guatemala $^{5}$ & 5,08 & 8,91 & 6,33 & 5,85 & 9,23 & 8,57 & 5,79 & 8,75 \\
\hline
\end{tabular}

Todos datos obtenidos del Índice de Precios al Consumidor interanual y acumulada que presenta cada fuente oficial.

1. Fuente. Banco Central de Nicaragua. http://www.bcn.gob.ni/index.asp

${ }^{2}$. Fuente. Banco Central de Costa Rica. http://indicadoreseconomicos.bccr.fi.cr/indicadoreseconomicos/

Cuadros

3. Fuente. Banco Central de Honduras. http://www.bch.hn/download/ipc_historico

${ }^{4}$. Fuente. Banco Central de Reserva de El Salvador. http://www.bcr.gob.sv/estadisticas/sr_precios. html

5. Fuente. Banco de Guatemala. http://www.banguat.gob.gt/inc/ver.asp?id=/estaeco/sr/ sr002\&e $=1780$

14 Cumbre de Jefes de Estado y Gobierno de Iberoamérica de 2007, Daniel realizó un discurso contra el imperialismo norteamericano y acusó al gobierno español liderado por Rodríguez Zapatero de representar los intereses del norte, a la par que propuso la creación de una organización de Estados Americanos ajena a la OEA liderada por Venezuela, Bolivia, Ecuador y Nicaragua.

15 Los nuevos países "amigos" del gobierno son Venezuela, Libia (donde el embajador de Nicaragua es el hijo del Presidente), Cuba, Irán, Bolivia y Ecuador.

16 Cabe señalar en esta dirección el pobre desempeño del Plan de Inversión Pública (PIP) que se planeó para el año 2007, del que sólo se ejecutó un $60 \%$ del gasto previsto a mediados de diciembre -extraído de la revista electrónica Confidencial, en el número de diciembre 2007.

17 Extraído de la revista electrónica Confidencial, en el número de diciembre 2007. 
En este contexto pareciera que la esperanza del "despegue" económico prometido pasó por "esperar" iniciativas comerciales del gobierno venezolano en el marco del ALBA y por la promesa de construcción de infraestructuras petroleras de manos -también- de la administración de Hugo Chávez -quien anunció en diciembre del 2007 desde La Habana que invertiría 2.500 millones de dólares en la construcción de una refinería de crudo en Nicaragua.

Mientras, la vulnerabilidad del país ante los desastres naturales no cedió. ${ }^{18} \mathrm{~A}$ inicios de septiembre el Huracán Félix entró con fuerza por Sandy Bay y destrozó comarcas enteras de la Región Autónoma del Atlántico Norte y a mediados de octubre las lluvias crearon otro desastre en la zona norte del país; reactivando una vez más la cultura de la emergencia donde el rol de la cooperación internacional continuó siendo vital. ${ }^{19}$ Cabe señalar que estos desastres supusieron modificaciones ad hoc a los Presupuestos de 2008. Unos presupuestos que tuvieron muchas dificultades para aprobarse en 2007 debido a la frágil situación que el FSLN tiene en la Asamblea Nacional, cuestión que merece la atención del próximo epígrafe.

\section{III. ¿CUÁN FRÁGIL ES LA POSICIÓN DEL FSLN EN LA ASAMBLEA NACIONAL?}

Al finalizar el primer epígrafe se hizo referencia a la ceremonia de toma de posesión del cargo de Presidente de la República por parte de Daniel Ortega. En ese párrafo se señaló la presencia de diversos Jefes de Estado, pero en el Estadio donde se realizó la ceremonia además de dichas autoridades estaba también un ex manadatario que no pasó inadvertido. Se trataba del ex presidente y reo Arnoldo Alemán. Este hecho señalaba que El Pacto (EP) entre el caudillo liberal y sandinista se mantenía firme. Este hecho no era baladí, pues del "buen entendimiento" entre los dos caudillos dependía tanto la capacidad del FSLN de aprobar leyes en la Asamblea Nacional, ${ }^{20}$ como la situación legal de Alemán. ${ }^{21}$

18 Nicaragua ha sido afectada por fenómenos naturales de diferente tipo en su historia reciente. Además de los fenómenos de plagas y sequías e inundaciones causadas por el Niño y la Niña (fenómenos del cambio climático) ha sufrido terremotos en 1931, 1968 y 1972 en Managua y en 2000 en Masaya y el tsunami en la costa del Océano Pacífico en 1992. Ha vivido huracanes como el Juana en 1988, el César en 1996, el Mitch en 1998 y las tormentas tropicales Brett y Gert en 1993. Y también ha sufrido las consecuencias de erupciones volcánicas del Cerro Negro en 1992 y 1995. Según datos de Defensa Civil, todos estos desastres naturales han afectado directamente a más de la mitad de la población (PNUD, 2007).

19 Es preciso notar que Nicaragua ha sido hasta la fecha uno de los países en vías de desarrollo con mayor volumen de ayuda internacional per cápita del mundo. Por ejemplo, en 2004 esta ayuda fue de US\$ 1.232 millones comprometidos, lo que significó una transferencia de US\$229,2 per cápita en cooperación, que representó el 27,1\% del PIB, según datos de la Organización de Cooperación y Desarrollo Económicos-Comité de Asistencia para el Desarrollo (PNUD, 2007).

20 El número de diputados de cada partido en la Asamblea Nacional puede verse en la Tabla 2 que se presenta en el anexo. Cabe señalar también que el FSLN controló durante la legislatura de 2007 la Presidencia de la Asamblea Nacional y la Segunda Secretaría; el PLC con la Segunda Vicepresidencia y la Primera Secretaría, así como la presidencia de las Comisiones de Economía, Justicia, Defensa y Gobernación.

21 Cabe señalar que durante el año 2007 el FSLN pretendió cambiar el status penal del caudillo liberal, primero otorgándole "país por cárcel”, y luego debatiendo su amnistía, durante el mes de agosto, en la misma Asamblea Nacional. 
Con todo, el mantenimiento de la alianza entre el PLC y el FSLN fue la incógnita de la legislatura debido a que las primeras reacciones del candidato a la Presidencia de la República del PLC, José Rizo, fueron de crítica hacia Arnoldo Alemán por la derrota electoral que sufrió su partido. Rizo dijo ${ }^{22}$ que fue un error del PLC haber pactado con el FSLN la reforma de la Ley Electoral y que "también fue un gran error haber promovido la creación de la ALN, al ignorar la realidad de ese 35\% y la fortaleza del FSLN". Agregó que la siguiente tarea de su partido era modernizarlo, "terminar con El Pacto, combatir la corrupción, promover la no reelección absoluta y trabajar por un liberalismo renovado". Rizo lamentó que el ex presidente Alemán ostentara el control del PLC, hecho que se demostró durante todo el año a través del control que el caudillo liberal tenía sobre la mayoría de los diputados del PLC.

El mantenimiento de EP supuso que durante toda la legislatura se mantuviera en la Asamblea Nacional una dinámica partidaria con coaliciones sin concordancia ideológica -tal como expusieron los líderes del MRS el 2 de julio de $2007^{23}$ al declarar que veían posible un acuerdo con ALN, pero nunca con el PLC ni el FSLN. Sin embargo, se vio también como algunos miembros del PLC se separaban de la línea que postulaba Alemán y se acercaban a posiciones próximas a la ALN; a la vez que dentro de ALN aparecía un grupo disidente de cuatro diputados que junto con los dos que mantenía el MRS crearon la llamada Bancada por la Unidad. Esta fluidez en las lealtades de los diputados opositores generó una gran incertidumbre a la hora de aprobar leyes en la Asamblea Nacional. ${ }^{24}$

De la práctica legislativa de 2007 se pudo percibir diversas dinámicas en la creación de mayorías. Por un lado hubo votaciones en las que los proyectos de ley fueron votados por todos los grupos parlamentarios, tal como ocurrió con la aprobación de leyes de gran relevancia. ${ }^{25}$ Por otro lado, se pudo ver la aprobación de leyes a pesar del voto en contra del MRS (tal como ocurrió con la reforma del Código Penal, Ley $\mathrm{N}^{\mathrm{o}}$ 641, en el que se penalizó el aborto terapéutico) y del rechazo de los legisladores del ALN y del mismo MRS -tal como ocurrió con las leyes N ${ }^{\circ} 612$ Ley de Reforma y Adición a la Ley $N^{\circ} 290$, Ley de Organización, Competencia y Procedimientos del Poder Ejecutivo ${ }^{26}$ y la No 615, Ley Anual de Presupuesto General de la República 2007. ${ }^{27}$ Y finalmente también aconteció el fenómeno de que toda la oposición votaba unida (bajo el nombre de Bloque Opositor) y frustraba iniciativas del FSLN.

Las citas entrecomilladas están extraídas de la revista electrónica Confidencial, del número posterior a las elecciones de noviembre de 2006.

23 Ese día se celebraba el primer aniversario del fallecimiento de Herty Lewites.

24 La producción legislativa realizada durante el año 2007 fue la siguiente: 33 leyes aprobadas en lo particular, tres leyes aprobadas en lo general, cinco dictámenes desfavorables, 40 decretos, 287 personalidades jurídicas, un veto presidencial aceptado y cuatro vetos presidenciales rechazados.

25 Estas leyes fueron las: Nº 610 Ley de Reforma a la Ley Marco para la Estabilidad y Gobernabilidad del país, No 617 Código Penal Militar, Nº 621 Ley de Acceso a la Información Pública, Nº 625 Ley del Salario Mínimo, No 627 Ley de Reformas y Adiciones a la Ley de Estabilidad Energética, y la Nº 640 Ley Creadora del Banco de Fomento a la Producción.

26 Aprobada el 23 de enero y publicado en La Gaceta.

27 Aprobada el 6 de marzo y publicada en La Gaceta No 56 del 20 de marzo. 
Este último caso fue especialmente notorio con la Ley $\mathrm{N}^{\circ} 630$ de Reforma y Adiciones al art. 11 de la Ley $N^{\circ} 290$ de Organización, Competencia y Procedimientos del Poder Ejecutivo, que si bien fue aprobada el 6 de septiembre, posteriormente -el 24 de septiembre- todos los diputados de la oposición presentaron un veto parcial (que fue exitoso al juntar 51 votos en contra) derogando la vinculación entre los Consejos del Poder Ciudadano y el Poder Ejecutivo. Con este veto parcial se frustró -en un inicio- la propuesta sandinista de crear un entramado nacional de instituciones de "participación directa" llamadas Consejos de Participación Ciudadana (CPC), semejantes a los antiguos Comités de Defensa Sandinistas de los años ochenta. ${ }^{28}$ Se expone que se frustró "en un inicio" porque ante el veto legislativo que se presentó en la Asamblea Nacional el FSLN interpuso, por un lado, un recurso de amparo contra dicho veto ante la Corte Suprema de Justicia y, por otro, el gobierno publicó el 29 de noviembre un Decreto Presidencial en que se comunicaba la creación de los CPC como una estructura gubernamental integrada al Consejo Nacional de Planificación Económica y Social (CONPES). Esta medida fue contestada inmediatamente por la oposición, haciéndose un gran eco en los medios de comunicación y en la opinión pública. ${ }^{29}$ Esta medida -la aprobación de los CPC por Decreto Presidencial- fue un punto de inflexión que cambió la dinámica legislativa.

De lo expuesto se puede interpretar que la mayoría de la legislación aprobada hasta 2007 se elaboró a través de coaliciones legislativas no conectadas ideológicamente (donde primó más la lógica "pacto vs. antipacto" que la de "izquierda vs. derecha"), y con la voluntad por parte del Ejecutivo de buscar un amplio consenso que les otorgara más apoyo que la fidelización de "coaliciones vencedoras mínimas". Este paisaje, sin embargo, cambió a finales de año con el ya citado conflicto de la creación por Decreto Presidencial de los Consejos de Participación Ciudadana, por lo que la legislatura de 2008 se prevé mucho más conflictiva que la que se analiza en este texto.

\section{UNA NUEVA CORRELACIÓN DE FUERZAS: ¿OTRA REFORMA CONSTITUCIONAL?}

En Nicaragua la normativa electoral y la misma Constitución han sido variables dependientes de las coyunturas e intereses de los actores políticos mayoritarios y no una variable

28 Los CPC pretenden crear una red de participación a nivel comarcal, barrial, municipal, departamental, regional y nacional; siendo la cúpula un consejo de representación conformado por unas 200 personas, con 16 coordinadores en diversas áreas temáticas (salud, educación, medio ambiente, transporte, desarrollo rural, caminos, cultura, deportes, comunicación y propaganda, derechos humanos, seguridad ciudadana, derechos de la mujer, derechos de jóvenes y niños, derechos de adultos mayores, propuestas hacia los gobiernos locales y programas de autoempleo). Cada CPC debe estar integrado por lo menos por 100 personas. La máxima autoridad de los CPC, nombrada por el Presidente Ortega, fue su esposa Rosario Murillo. Si bien aún no se han puesto en práctica los CPC se teme que éstos sean plataformas de movilización afines al FSLN desde las cuales el partido fidelice su militancia a cambio de la distribución de recursos.

29 El día 9 de septiembre hubo una marcha, convocada por el Movimiento por Nicaragua, contra la creación de los CPCs. 
independiente capaz de configurar un escenario político que a fuerza de voto estratégico y hábitos haya ido cristalizándose. ${ }^{30}$

En esta lógica el año 2007, y con la llegada de una nueva administración, las veleidades reformistas se volvieron a activar. ${ }^{31}$ La administración de Ortega "resucitó" un proyecto de reforma constitucional que estaba presente-pero sin haberse hecho efectivo-desde 2005. Se trataba de la Ley 520 que introduce una nueva redacción de los artículos 138.4, 138.9 y 150.6 de la Constitución vigente. La reforma de los artículos referidos se proyectaba sobre aspectos fundamentales de las relaciones entre el Poder Ejecutivo y el Poder Legislativo, otorgando a la Asamblea Nacional la capacidad de nombrar y ratificar altos funcionarios del Poder Ejecutivo, creando nuevos mecanismos de control parlamentario sobre el gobierno -tales como la solicitud de informes, la comparecencia personal e interpelación y la destitución (Vintró y Álvarez, en prensa).

Pero esta reforma -elaborada en 2005- no entró en vigor en su momento debido a que se aplazó su efectividad con la aprobación el 20 de octubre de 2005 de la Ley 558 (llamada Ley Marco para la Estabilidad y Gobernabilidad) hasta el 20 de enero del 2007.

Así, con la llegada de Daniel Ortega a la Presidencia reapareció en la agenda política la Ley 520. Pero rápidamente la Asamblea Nacional propuso y aprobó otra ley (la Ley 610 de reforma a la Ley Marco) que prorrogó una vez más la entrada en vigencia de la reforma constitucional hasta el 20 de enero del 2008. Sin embargo, en el mes de diciembre de 2007 el debate político se centró en la amenaza de todos los grupos parlamentarios opositores -a través del Bloque Opositor - de activar la Ley 520 y limitar con dicha reforma constitucional la capacidad de maniobra del presidente. ${ }^{32}$

A este debate y a las amenazas de la oposición se adelantó la Corte Suprema de Justicia que, con una sentencia del 10 de enero de 2008, declaró inconstitucionales las Leyes 558 y 610 y, por lo tanto, desde dicha fecha la Ley 520 de reforma constitucional se puso en vigor y la administración Ortega empezó a operar en un entorno político mucho más exigente y con mayores constricciones del que lo hizo en 2007.

$30 \quad$ Puede constatarse cómo la apertura de espacios electorales de 1984 y 1990 fue debida a la presión ejercida por la administración norteamericana, a la agresión bélica liderada por la Contra y a los acuerdos regionales de Esquipulas II con sus consiguientes rondas de negociación (llamadas Comisiones Nacionales de Reconciliación) entre la administración sandinista y la “oposición desleal” (Martí,1997). Pero también podría exponerse que las reformas a la Constitución acaecidas en 1995, 1997, 2000 y 2005 han sido fruto de negociaciones forzadas desde intereses ajenos a la institucionalidad con el objeto de abrir o cerrar espacios políticos en función de las estrategias "cortoplacistas" de las formaciones partidarias mayoritarias (Close y Martí, en prensa).

31 En la Asamblea Nacional se creó una Comisión Especial que procedió a elaborar un anteproyecto de reformas constitucionales donde las fórmulas "parlamentarizantes" tienen una notable acogida entre los líderes del FSLN y del PLC.

32 Información extraída del periódico La Prensa, del día 2 de enero de 2008. 


\section{V. ¿QUÉ TIPO DE PARTIDO SE HA CONVERTIDO EL FSLN? YQUÉ PUEDE ESPERARSE DE LA SEGUNDA ADMINISTRACIÓN ORTEGA?}

Se ha escrito bastante sobre qué cambios podían acontecer con el regreso del FSLN al poder, sin embargo antes de hablar de ello es importante exponer una nota sobre las transformaciones acontecidas en el mismo FSLN durante sus 16 años en la oposición y, en definitiva, sobre qué tipo de partido es el que ha llegado a ejercer el poder en Nicaragua en 2007.

Respecto a la dimensión organizativa, desde 1990 el FSLN gestionó el "consenso organizativo" exclusivamente a través de la "salida" y la "lealtad", pero nunca a través de la "voz", hecho que supuso una dinámica constante de enfrentamientos personales. Sin embargo, con ello el FSLN fue disciplinando la organización bajo una conducción tan centralizada que, desde 1996, desembocó en un liderazgo personal: el de Daniel Ortega (y que, desde finales de 2006 y crecientemente en 2007 se le ha sumado el de su cónyuge Rosario Murillo). En esta dirección no deja de sorprender que el discurso de aceptación de "la democracia" como sistema político que abanderó el FSLN después de los resultados de 1990 (y que se esgrimió durante los años noventa como la "gran conquista" nacional de la revolución sandinista) coexistió siempre con una reservada disposición a aplicarla dentro del mismo FSLN, manteniendo incluso "tics" organizativos de disciplina y lealtad heredados de la época de la clandestinidad.

Estos "tics" organizativos han supuesto un estigma que, tal como expone Panebianco (1990) en su "teoría genética" de las organizaciones partidarias, ha acompañado a la formación durante toda su historia. Sin embargo, más allá de eso las características organizativas del FSLN han cambiado profundamente desde la derrota electoral de 1990 hasta la victoria de 2006 y el ejercicio del poder en 2007. El aparato burocrático casi para-estatal de Partido Vanguardia que dirigía la revolución durante los años ochenta tuvo que desmantelarse con la pérdida del poder y, con ella, desaparecieron los recursos públicos. Fue entonces cuando el FSLN tuvo que transformarse súbitamente.

En poco tiempo el FSLN se adaptó a un entorno donde los recursos de la organización dependían, sobre todo, de la capacidad de obtener (y retener) cargos institucionales, mayoritariamente electos (Santiuste, 2001). Ello supuso el "adelgazamiento" de la organización y la mutación de la naturaleza de sus cuadros políticos, que pasaron de ser gestores públicos o miembros de los cuerpos armados para convertirse en cargos electos, ya fueran diputados, alcaldes, concejales o miembros de organismos autónomos del Estado. A raíz de esa doble mutación -en el tamaño organizativo y en la naturaleza de sus cuadros-la máquina del FSLN se convirtió en una "pequeña" organización férreamente jerarquizada, con una importante implantación territorial en el país (aunque con una mayor presencia en el Pacífico que en el interior o el Atlántico) y con la disposición de importantes recursos materiales, destacando la propiedad de canales de televisión y de radio. ${ }^{33}$

33 Actualmente, una forma de obtener la información "oficial" del FSLN es a través de la página electrónica http:/ / www.lavozdelsandinismo.com/ en la que, además, aparecen los vínculos con otros "medios afines". 
De lo expuesto y utilizando las categorías de análisis del poder organizativo (Panebianco 1990) es posible afirmar que si bien antes de la toma del poder en 1979 el FSLN encajaba perfectamente a la tipología de un actor político que respondía a las características del modelo organizativo de sistema de solidaridad, después de ejercer el poder durante una década y de su paso por la oposición éste incorporó rápidamente los caracteres propios del modelo de sistema de intereses en tanto que la organización pasó de ser una "variable dependiente" de los fines (la victoria revolucionaria y la transformación de la sociedad) a convertirse casi en un "fin en sí misma", adaptada completamente a una multitud de demandas provenientes de diferentes sectores internos y a la voluntad de retener (en la década de los ochenta) y alcanzar (después de 1990) el poder. Con ello, el FSLN, adoptó características de una organización completamente institucionalizada, donde las actividades destinadas a asegurar su supervivencia e incrementar las cuotas del poder se impusieron a cualquier otra lógica. Fruto de lo expuesto surgió la necesidad de ofrecer incentivos selectivos para la satisfacción de demandas individuales de unos miembros que ya no sólo respondían al concepto de "militante creyente".

En esta misma lógica, cabe observar cómo el FSLN ha podido manejar a discreción un recurso simbólico tan importante como es el mismo discurso partidario. Sobre ello es gráfico ver cómo el FSLN ha tenido la capacidad de continuar elaborando, desde 1990, una retórica radical con un desempeño práctico absolutamente pragmático y con fines esencialmente tácticos. Una muestra gráfica de esta "capacidad" fue la distancia que medió entre su discurso contra el CAFTA-DR ante sus bases electorales y su misma aprobación en la Asamblea Nacional; o el apoyo de una legislación contra el aborto terapéutico (de la mano de la Iglesia Católica) a la par que se sumaba a las declaraciones de las "internacionales partidarias" más izquierdistas de la región, y se alineaba internacionalmente con Hugo Chávez, Evo Morales o Muhamar el Kadaffi. Así el FSLN se centró progresivamente en la exaltación de símbolos patrios y en el despliegue de slogans esotéricos y espirituales, que han tenido su máxima expresión con la "oficialidad" de la fiesta de la Purísima en 2007.34

Respecto a la estrategia seguida por el FSLN para la "reconquista" del poder es preciso señalar que éste ha tenido la capacidad de mantener un notable desempeño en el juego electoral (tal como se observa en la Tabla 1 del anexo) y una excelente capacidad de negociación con el resto de las elites políticas del país con el objetivo de una acumulación paulatina de cuotas de poder, y con un riesgo limitado de rebelión partidaria.

Por ello resulta asombrosa la habilidad que ha tenido la dirección del FSLN para disciplinar sus bases bajo un liderazgo personalista y para apropiarse de los recursos simbólicos históricos del sandinismo. Esta libertad de movimientos -totalmente insospechada hace dos décadas- es difícil de comprender sin tener en cuenta el continuado proceso de construcción, por parte de la organización, de un liderazgo excluyente -y no necesariamente carismático- que ha suplantado a la antigua dirección colegiada.

34 El día 28 de noviembre la Primera Dama y portavoz de la Presidencia, anunció que el Presidente "instruyó a todas las dependencias del gobierno a celebrar la Purísima" y declaró a la Virgen María la "María de Nicaragua". 
Este proceso de mutación acelerado ha convertido al FSLN en un partido que responde, en gran medida, al modelo "profesional electoral" con elementos caudillistas y con tácticas propias del "partido cártel". Una formación "profesional electoral" porque, tal como hemos visto, el FSLN ha acabado por ser una organización pequeña y centralizada que prioriza la competición electoral, y caudillista porque sus máximos dirigentes -Daniel Ortega y desde hace poco su esposa- controlan con discreción los recursos institucionales y organizativos (Martí y Santiuste, 2006). Sin embargo, además de estas características el FSLN también ha incorporado elementos propios de un "partido cártel", tal como lo ejemplifica la gran autonomía que ha gozado su líder, y el progresivo debilitamiento de la posición de los militantes y activistas en las tareas partidarias. Pero lo que más acerca al FSLN a la caracterización de "partido cártel" ha sido su beligerante estrategia para obtener, a través de negociaciones con el resto de formaciones políticas relevantes, recursos públicos que le supusieran una reducción de costes ante posibles derrotas electorales y el manejo de las reglas institucionales a su favor -donde el EP ha sido su máxima expresión.

Por todo ello cabe concluir que la adaptación del FSLN ha sido tan "exitosa" y de tal envergadura que puede afirmarse que el Frente, que ha vuelto al poder en 2007 por la vía electoral -más allá de la figura de Ortega-, casi no mantiene elementos en común con el que, hace tres décadas, lideró una insurrección popular bajo la premisa de una transformación revolucionaria. Siguiendo dicho razonamiento es plausible entender la continuidad con las anteriores administraciones liberales en cuanto a las políticas macroeconómicas y de desarrollo. ${ }^{35} \mathrm{Y}$ si bien cabe señalar nuevas tendencias en cuanto a la implementación de políticas sociales focalizadas, la retórica del Jefe de Estado en foros internacionales y las alianzas geopolíticas, pareciera que durante los primeros pasos de la segunda administración Ortega el mayor cambio experimentado ha sido la circulación de los cargos públicos y el "estilo" del gobierno.

\section{REFERENCIAS}

Carmines, Edward G. y Wagner, Michael W. 2006. "Political Issues and Party Alignements: Assessing the Issue Evolution Perspectives" en: Annual Review of Political Science 9: 67-81.

Carmines, Edward G., McIver, John P., Stimson, James A. 1987. “Unrealized Partisanship: A Theory of Dealignment" en: The Journal of Politics 49(2): 376-400.

Close, David. 2004. "President Bolaños Runs a Reverse, or How Arnoldo Alemán Wound Up in Prison" en: Close, David y Deonandan, Kalowatie eds. 2004. Undoing Democracy. The Politics of Electoral Caudillismo. NY: Lexington Books.

Close, David y Salvador Martí (en prensa). The Sandinistas and Nicaraguan Politics since 1979. Palgrave, NY.

Dye, David R. 2001. Retazos de democracia. La política nicaragüense diez años después de la derrota. [En línea] $<$ www.hemisphereinitiatives.org>

2004. La Democracia a la deriva: La política Caudillista de Nicaragua. [En línea] <www. hemisphereinitiatives.org $>$.

Key, V.O. 1995. "A Theory of Critical Elections" en: Journal of Politics 17: 1-18.

35 En esta dirección hay intelectuales que presentan la hipótesis de que la administración de 2007 inaugura un período de "sandinismo de derechas" (Torres-Rivas, 2007). 
1964. "The Party Battle, 1896-1969" en: Politics, Parties and Pressure Groups. NY: Thomas Y. Crowell Company.

Martí i Puig, Salvador. 1997. Nicaragua 1977-1996. La revolución enredada. Madrid: Libros de la Catarata. . 2008. "El regreso del FSLN al poder: ¿Es posible hablar de realineamiento electoral en Nicaragua?”. Revista Política y Gobierno, XIV (1).

Martí i Puig, Salvador y Salvador Santiuste. 2006. “El FSLN: de guerrilla a oposición negociadora”, en La izquierda revolucionaria en Centroamérica. De la lucha armada a la participación electoral. Editado por S. Martí i Puig y C. Figueroa. Madrid: Libros de la Catarata.

Nitlápan-Envío. 2006. "Así está el escenario al iniciarse la campaña electoral". Revista Envío No 293. [En línea] <www.envio.com.ni>.

Ortega Hegg, Manuel. 2006. "Los resultados electorales del 2006 en Nicaragua”. [En línea] <www.boelllatinoamericana.org $>$.

2007. "Nicaragua 2007: El regreso del FSLN al poder". Revista de Ciencia Política 27 (E.E.): 206-219.

Panebianco, Angelo. 1900. Modelos de partido. Editorial Alianza, Madrid.

Pérez Baltodano, Andrés. 2005. "Nicaragua: un experimento democrático en agonía". Revista Nueva Sociedad 199. [En línea] <www.nuso.org/revista.php?n=204>.

2006. "La visión no conflictiva de la política". [En línea] <www.envio.com.ni>.

Programa de Naciones Unidas para el Desarrollo (PNUD). 2007. Evaluación de los resultados del desarrollo. Nicaragua. PNUD: NY.

Santiuste Cué, Salvador. 2001. "La incompleta transformación del FSLN", América Latina Hoy 27: 75-98.

Spalding, Rose (en prensa). "The Economic Impact of Public Policies", en: Close, David y Salvador Martí (en prensa), The Sandinistas and Nicaraguan Politics since 1979. Palgrave, NY.

Torres-Rivas, Edelberto. 2007. “Nicaragua: el retorno de un sandinismo transfigurado", en Nueva Sociedad 207: 4-10.

Vintró, Joan y Álvarez, Gabriel (en prensa). "Constitutions and Institutional Change", en The Sandinistas and Nicaraguan Politics since 1979, editado por D, Close y S. Martí . NY: Palgrave,

Warren, Millar. 1991. "Party Identification, Realignment, and Party Voting: Back to the Basics". The Amercian Political Science Review 85(2): 557-568.

\section{RECURSOS ELECTRÓNICOS UTILIZADOS}

www.envio.org.ni

www.elnuevodiario.org,ni

www.laprensa.org.ni

www.confidencial.org.ni

www.presidencia.gob.ni

www.asamblea.gob.ni

www.lavozdelsandinismo.com/ 


\section{ANEXOS}

Tabla 1: Resultados de las elecciones presidenciales (en porcentajes) y escaños en las legislativas (1990-2006)

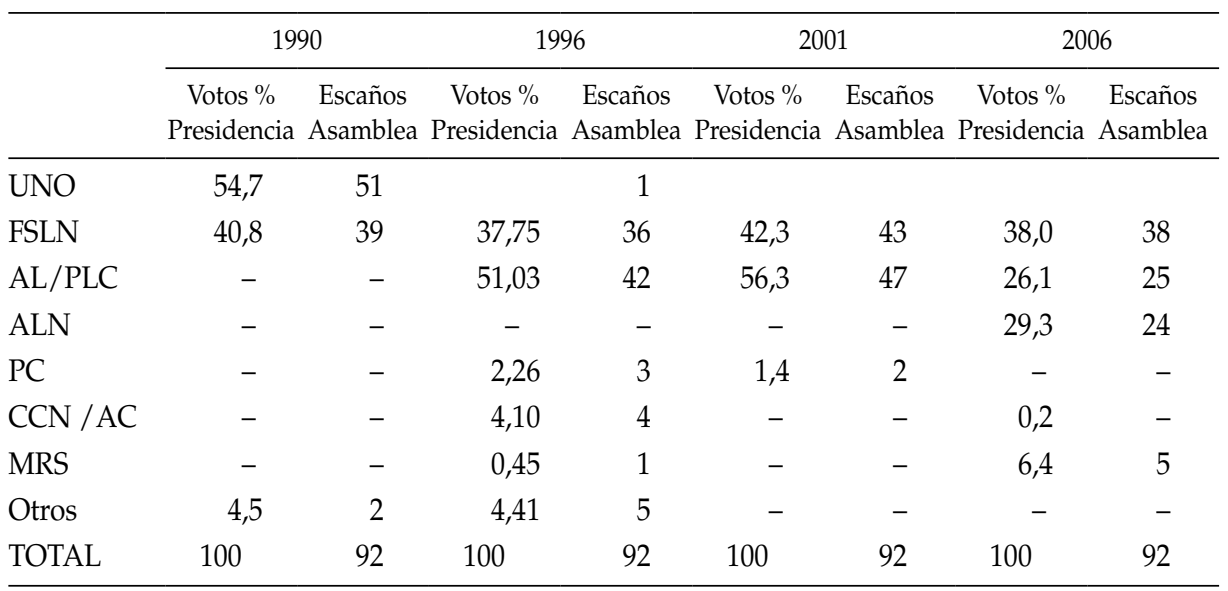

Fuente: Elaboración propia a partir de datos del Consejo Supremo Electoral.

Tabla 2: Asignación de diputados por partido de las elecciones de 2006

\begin{tabular}{lccccc}
\hline Departamentos & Escaños & PLC & FSLN & ALN & MRS \\
\hline Lista Nacional & 20 & 6 & 8 & 5 & 1 \\
Nueva Segovia & 2 & 1 & 1 & 0 & 0 \\
Madriz & 2 & 1 & 1 & 0 & 0 \\
Estelí & 3 & 1 & 2 & 0 & 0 \\
Chinandega & 6 & 0 & 4 & 2 & 0 \\
León & 6 & 1 & 3 & 2 & 0 \\
Managua & 19 & 4 & 7 & 5 & 3 \\
Masaya & 4 & 1 & 2 & 1 & 0 \\
Carazo & 3 & 0 & 1 & 1 & 1 \\
Granada & 3 & 0 & 1 & 2 & 0 \\
Rivas & 2 & 0 & 1 & 1 & 0 \\
Chontales & 3 & 1 & 1 & 1 & 0 \\
Boaco & 2 & 1 & 1 & 0 & 0 \\
Matagalpa & 6 & 3 & 2 & 1 & 0 \\
Jinotega & 3 & 2 & 1 & 0 & 0 \\
RAAN & 3 & 1 & 2 & 0 & 0 \\
RAAS & 2 & 1 & 0 & 1 & 0 \\
Río San Juan & 1 & 1 & 0 & 0 & 0 \\
TOTAL & 90 & 25 & 38 & 22 & 5 \\
\hline
\end{tabular}

Fuente: Elaboración propia a partir de datos del Consejo Supremo Electoral. 
Tabla 3: Posiciones de los partidos por Departamentos en las elecciones Presidenciales de 2006

\begin{tabular}{|c|c|c|c|}
\hline & FSLN & PLC & ALN \\
\hline Ganador & $\begin{array}{l}\text { Nueva Segovia, Madriz, } \\
\text { Estelí, Chinandega, } \\
\text { León, Managua, Carazo, } \\
\text { Matagalpa, RAAN } \\
\text { (pacto con Yatama) }\end{array}$ & $\begin{array}{l}\text { Chontales, Boaco, } \\
\text { Jinotega, RAAS y RSJ }\end{array}$ & Masaya, Granada, Rivas \\
\hline Segundo puesto & $\begin{array}{l}\text { Masaya, Granada, Rivas, } \\
\text { Boaco, Jinotega, Río San } \\
\text { Juan }\end{array}$ & $\begin{array}{l}\text { Nueva Segovia, Madriz, } \\
\text { Estelí, Matagalpa, } \\
\text { RAAN }\end{array}$ & $\begin{array}{l}\text { Chinandega, León, } \\
\text { Managua, Carazo, } \\
\text { RAAS y Chontales }\end{array}$ \\
\hline Tercer Puesto & Chontales y la RAAS & $\begin{array}{l}\text { Chinandega, León, } \\
\text { Managua, Masaya, } \\
\text { Carazo, Granada, Rivas }\end{array}$ & $\begin{array}{l}\text { Nueva Segovia, Madriz, } \\
\text { Estelí, Matagalpa, } \\
\text { Río San Juan, RAAN, } \\
\text { Jinotega }\end{array}$ \\
\hline
\end{tabular}

Gráfico 1: Cleavages y formaciones políticas en las elecciones de 2006

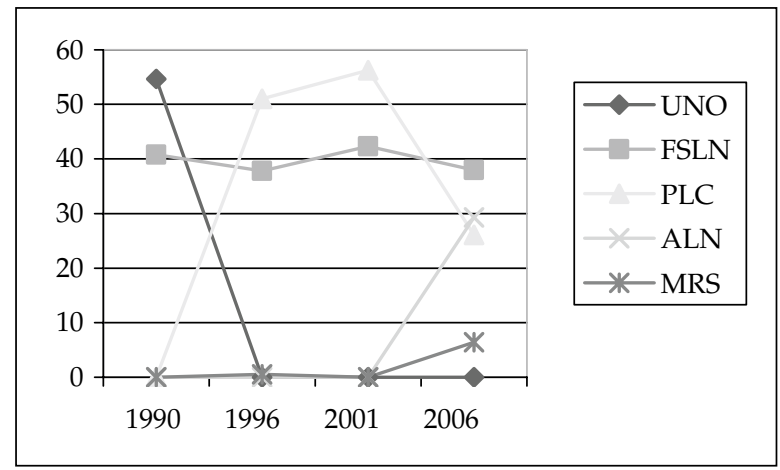

Salvador Martí i Puig es doctor en Ciencia Política por la Universidad Autónoma de Barcelona y actualmente es profesor titular en la Universidad de Salamanca y del IBEI-Barcelona. Ha colaborado como consultor en el PNUD en temas de desarrollo y gobernabilidad. Es autor de diversos libros y artículos para revistas especializadas sobre procesos de cambio político en Mesoamérica también sobre acción colectiva. Sus últimos libros son Pueblos indígenas y Política en América Latina (Bellaterra-Cidob, 2007) y, junto con David Close está elaborando la obra titulada The Sandinistas and Nicaraguan Politics since 1979. (Palgrave). E-Mail: smarti@usal.es 
